\title{
$\beta$-Arrestin2 mediates progression of murine primary myelofibrosis
}

\author{
Lindsay A.M. Rein, ${ }^{1}$ James W. Wisler, ${ }^{2}$ Jihee Kim, ${ }^{3}$ Barbara Theriot, ${ }^{3}$ LiYin Huang, ${ }^{3}$ Trevor Price, ${ }^{1}$ \\ Haeyoon Yang, ${ }^{1}$ Minyong Chen, ${ }^{4}$ Wei Chen, ${ }^{4}$ Dorothy Sipkins, ${ }^{1}$ Yuri Fedoriw, ${ }^{5}$ Julia K.L. Walker, ${ }^{6}$ \\ Richard T. Premont, ${ }^{4}$ and Robert J. Lefkowitz ${ }^{7}$ \\ DDivision of Hematologic Malignancies and Cellular Therapy, ${ }^{2}$ Division of Cardiology, ${ }^{3}$ Department of Medicine, and \\ ${ }^{4}$ Division of Gastroenterology, Duke University, Durham, North Carolina, USA. ${ }^{5}$ Department of Pathology and Laboratory \\ Medicine, University of North Carolina, Chapel Hill, North Carolina, USA. ${ }^{6}$ Duke University School of Nursing and \\ 'Department of Medicine, Department of Biochemistry, and Howard Hughes Medical Institute, Duke University, Durham, \\ North Carolina, USA.
}

Primary myelofibrosis is a myeloproliferative neoplasm associated with significant morbidity and mortality, for which effective therapies are lacking. $\beta$-Arrestins are multifunctional adaptor proteins involved in developmental signaling pathways. One isoform, $\beta$-arrestin2 ( $\beta$ arr2), has been implicated in initiation and progression of chronic myeloid leukemia, another myeloproliferative neoplasm closely related to primary myelofibrosis. Accordingly, we investigated the relationship between $\beta$ arr2 and primary myelofibrosis. In a murine model of MPLW515L-mutant primary myelofibrosis, mice transplanted with donor $\beta$ arr2-knockout ( $\beta$ arr2 $\left.{ }^{-/-}\right)$hematopoietic stem cells infected with MPL-mutant retrovirus did not develop myelofibrosis, whereas controls uniformly succumbed to disease. Although transplanted $\beta$ arr2 ${ }^{-1-}$ cells homed properly to marrow, they did not repopulate long-term due to increased apoptosis and decreased self-renewal of $\beta$ arr $2^{-/-}$cells. In order to assess the effect of acute loss of $\beta$ arr 2 in established primary myelofibrosis in vivo, we utilized a tamoxifen-induced Cre-conditional $\beta$ arr2-knockout mouse. Mice that received Cre (+) donor cells and developed myelofibrosis had significantly improved survival compared with controls. These data indicate that lack of antiapoptotic $\beta$ arr2 mediates marrow failure of murine hematopoietic stem cells overexpressing MPLW515L. They also indicate that $\beta$ arr2 is necessary for progression of primary myelofibrosis, suggesting that it may serve as a novel therapeutic target in this disease.

Conflict of interest: The authors have declared that no conflict of interest exists.

Submitted: October 18, 2017 Accepted: November 1, 2017 Published: December 7, 2017

\section{Reference information:} JCI Insight. 2017;2(23):e98094. https://doi.org/10.1172/jci. insight.98094.

\section{Introduction}

Primary myelofibrosis (PMF) is a myeloproliferative neoplasm (MPN) of hematopoietic stem cells (HSCs) characterized by clonal proliferation of myeloid cells and secondary marrow fibrosis. PMF patients have significant disease-related morbidity (pancytopenia, hepatosplenomegaly, constitutional symptoms, weight loss) and a $15 \%-20 \%$ chance of transformation to acute leukemia, which is almost uniformly fatal despite aggressive management $(1,2)$. Current therapeutic options are limited and include JAK inhibitors; interferons; symptom-directed therapies, including steroids and cytoreductive agents; and allogeneic stem cell transplantation. Unfortunately, highly effective therapies for this progressive, debilitating disease are lacking and novel therapies are needed (3-5).

The gain-of-function mutation in JAK2, JAK2V617F, occurs in $45 \%-50 \%$ of PMF patients, while the myeloproliferative leukemia (MPL) gene mutation of the thrombopoietin receptor, MPLW515L, occurs in $5 \%-10 \%$ of PMF patients. Such mutations lead to enhanced signaling downstream of hematopoietic-specific cytokine receptors, including thrombopoietin. Interestingly, expression of both mutations in murine and human HSCs leads to cytokine-independent growth, with constitutive activation of downstream signaling pathways, including the JAK/STAT pathway (6-8). Agents targeting JAK2 have proven effective in patients with or without targeted mutations, suggesting that additional signaling pathways are involved in disease pathogenesis. Accordingly, alternative mechanisms of direct and indirect JAK/STAT activation have been described (9-11).

Recently, we investigated the role of $\beta$-arrestins in chronic myeloid leukemia (CML), another MPN of HSCs characterized by excess cell proliferation (12). $\beta$-Arrestin1 ( $\beta$ arr1) and $\beta$-arrestin2 ( $\beta$ arr2) are 
multifunctional adaptor proteins originally identified as negative regulators of G protein-mediated signaling. $G$ protein-coupled receptors bind extracellular ligands and activate second messenger-mediated intracellular signaling pathways, making them ideal drug targets in many diseases (13). $\beta$-Arrestins have also been identified as mediators of distinct signaling cascades independent of $G$ proteins (14). This work has led to the investigation of $\beta$-arrestins' role in cancers, including lung, colon, breast, and hematologic malignancies $(15,16)$.

Our recent work utilizing a mouse CML model demonstrated that lack of $\beta$ arr2 decreased incidence and hindered progression of disease due to altered Wnt/ $\beta$-catenin signaling. This study suggested that long-term HSC self-renewal is dependent on $\beta$ arr2-mediated signaling, especially under conditions of increased cellular stress (12). However, the role of $\beta$-arrestins in other MPNs remains to be characterized. Here, we examined the role of $\beta$-arrestins in the initiation of MPLW515L-mutant PMF. Additionally, using a conditional transgenic mouse model, we demonstrate that deleting $\beta$ arr 2 from established PMF mitigates the course of disease. Ultimately, we aimed to identify novel therapeutic targets in a disease for which effective therapies are severely lacking.

\section{Results}

Barr2 is necessary for development of PMF in a murine model of disease. To assess $\beta$-arrestins' role in PMF development, we utilized WT, $\beta$ arr1-knockout $\left(\beta a r r 1^{-/}\right)$, and $\beta$ arr2-knockout ( $\beta$ arr $\left.{ }^{-/-}\right)$KLS (c-Kit ${ }^{+}$, Sca- $1^{+}$, Lin $\left.{ }^{-}\right)$cells and standard retroviral transduction systems to model MPLW515L-mutant PMF $(17,18)$. Signs of murine PMF included leukocytosis, thrombocytosis, anemia, hepatosplenomegaly, and weight loss. To ensure that no baseline differences in global knockout mice were present, we characterized hematologic parameters, including white blood cells (WBCs), platelets, and hemoglobin, in these animals (Supplemental Figure 1A; supplemental material available online with this article; https://doi.org/10.1172/jci.insight.98094DS1). All hematologic parameters were phenotypically similar excluding WBCs, which were higher in WT mice compared with $\beta$ arr $1^{-/-}$and $\beta$ arr $2^{-/-}$mice. Despite being significantly increased, the average WBC count for WT mice remained within the normal range for mice on a C57BL/6J background. WBC counts for the $\beta$ arr $1^{-/}$and $\beta a r r 2^{-/-}$mice were similarly within the normal range for mice on a C57BL/6J background. The percentage of KLS cells harvested from marrow as well as liver and spleen size were similar across genotypes (Supplemental Figure 1, B and C).

To assess $\beta$-arrestin involvement in the development of PMF, donor WT, $\beta$ arr $1^{-/}$, and $\beta$ arr2 ${ }^{-/-} \mathrm{KLS}$ cells were harvested, infected with MPLW515L-GFP-expressing retrovirus, resorted for GFP expression by flow cytometry, and injected into lethally irradiated recipient mice (Supplemental Figure 2). Mice that received Barr $2^{-/-}$cells had dramatically increased median survival, with only 1 of 16 mice dead by the study endpoint compared with mice receiving WT or $\beta$ arr $1^{-/-}$cells (median survival 50 and 41 days) $(P<0.0001)$ (Figure 1A). The spleen-to-body ratio at death was larger in mice receiving WT or $\beta$ arr $1^{-{ }^{-}}$cells $(2.98 \%$ and $4.16 \%)$ compared with mice receiving $\beta$ arr $2^{-/-}$cells $(0.44 \%, P<0.0001$; Figure $1 \mathrm{~B})$. The liver-to-body ratio at death was also larger in mice receiving WT or $\beta$ arr $1^{-/-}$cells $(11.84 \%$ and $10.58 \%)$ compared with mice receiving Barr $2^{-/-}$cells $(4.09 \%, P<0.0001$; Figure 1B). Blood samples were obtained weekly from surviving mice for analysis. WBCs and platelets were lower over time in mice receiving $\beta$ arr $2^{-/}$cells versus mice receiving either WT or $\beta$ arr1 ${ }^{-/}$cells (WBCs, $P<0.01$ for interaction, $P<0.0001$ WT versus $\beta$ arr2 $2^{-/-}, P<0.0001$

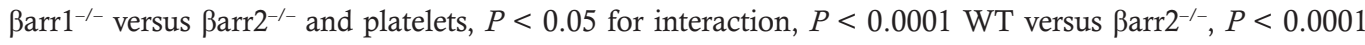
$\beta a r r 1^{-/-}$versus $\beta$ arr $\left.2^{-/}\right)$. Hemoglobin was also high over time in mice receiving $\beta$ arr $2^{-/-}$cells $(P<0.0001$ for interaction). Interestingly, mice that received $\beta$ arr $2^{-/}$cells had minimal donor cell engraftment over time versus mice receiving WT or $\beta$ arr $1^{-/-}$cells $\left(P<0.05\right.$ for interaction, $P<0.0001 \mathrm{WT}$ vs. $\beta$ arr $2^{-/}$and $\beta \operatorname{arr} 1^{-/-}$ vs. $\beta_{\text {arr }}{ }^{--}$) (Figure 1C).

These findings, taken together, indicate that mice receiving $\beta$ arr $2^{-/-}$cells do not develop PMF, due to lack of engraftment of donor cells, while mice receiving WT or $\beta$ arr1 $1^{-/}$cells engraft and develop disease. Histologic development of fibrosis in mice receiving WT or $\beta$ arr $1^{-/}$cells was consistent with this conclusion (Supplemental Figure 3).

Donor KLS cells from Barr2-/- mice home to marrow but fail to engraft and repopulate the marrow. To establish $\mathrm{PMF}$ in our mouse model, donor cells must home to marrow and engraft for long-term repopulation. These processes are regulated by common signaling mechanisms. Homing is rapid, occurs within hours of transplant, does not require cell division, and involves multiple cell types. Engraftment, both short and longterm, and subsequent marrow repopulation occur over weeks to months and require active cell division and 
A

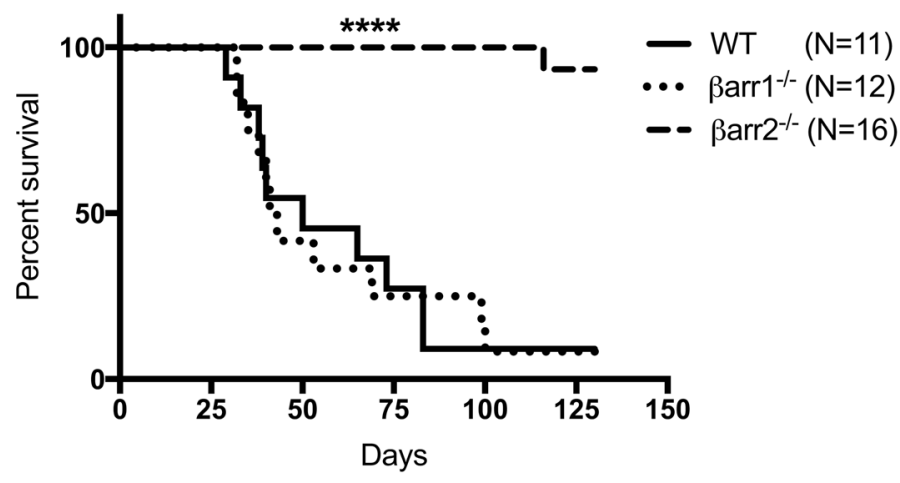

B

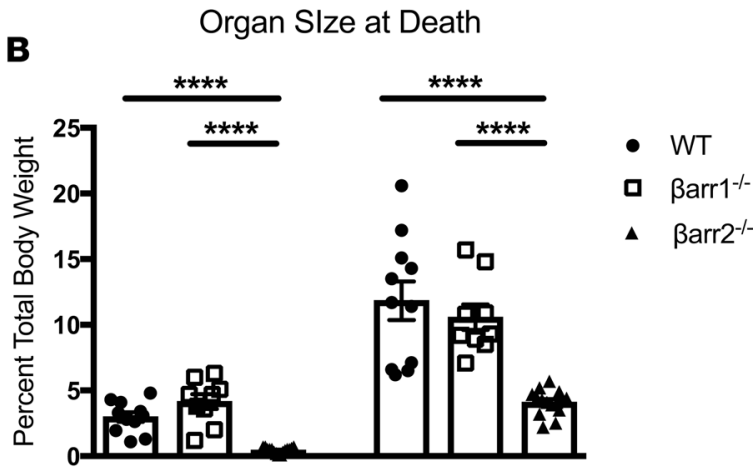

Spleen

Organ Slze at Death

Liver

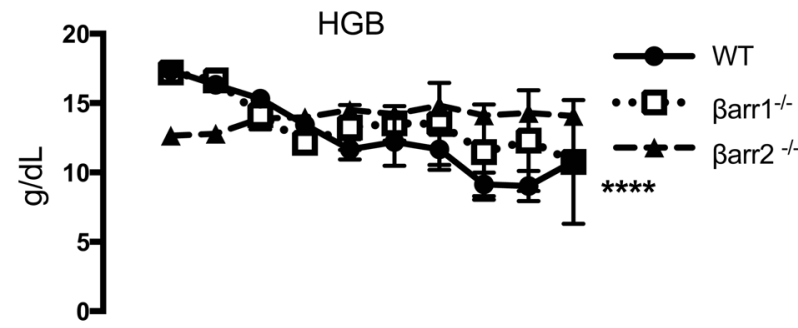

C
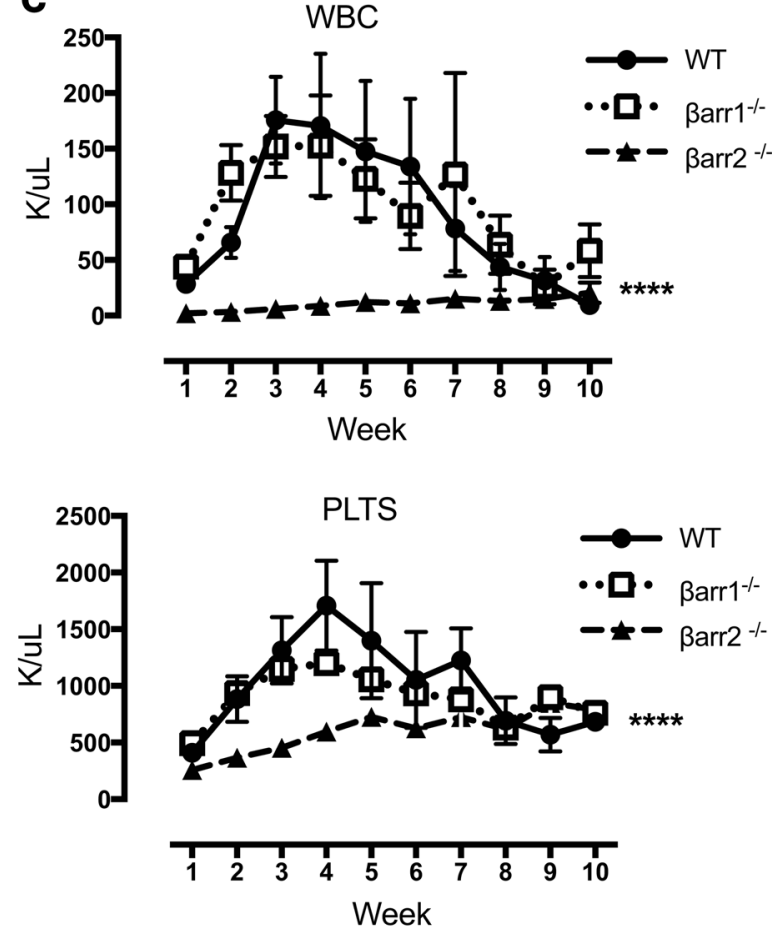
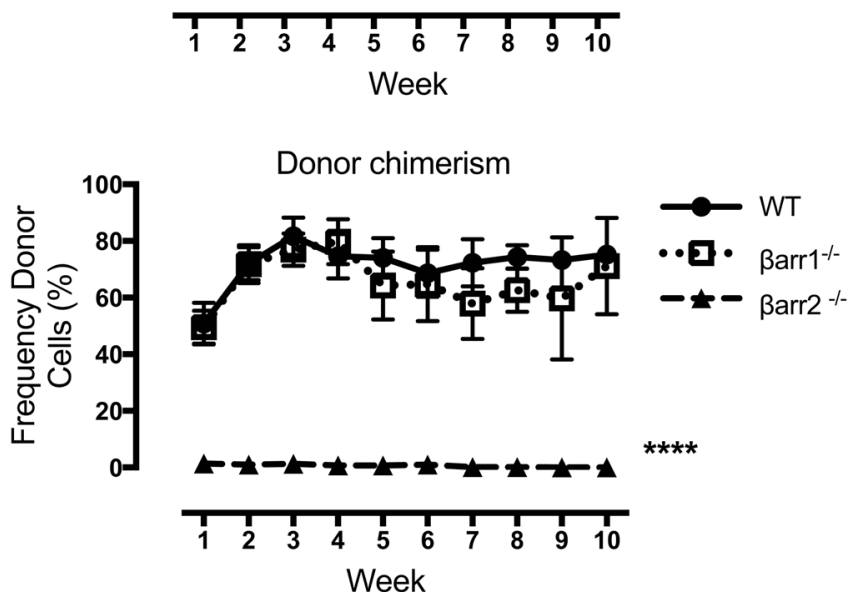

Figure 1. $\beta$-Arrestin2 is necessary for development of primary myelofibrosis in a murine disease model. WT, $\beta$-arrestin1-knockout ( $\beta$ arr1 $\left.{ }^{-/-}\right)$, and $\beta$-arrestin2-knockout ( $\beta$ arr2 ${ }^{--}$) KLS cells and a retroviral transduction system were used to model MPLW515L-mutant primary myelofibrosis (PMF). (A) Median survival of mice receiving MPLW515L-expressing $\beta$ arr $2^{-1-}$ donor cells is longer compared with WT or $\beta$ arr1 ${ }^{-/-}$cell recipients by log-rank Mantel-Cox test $\left.{ }^{* * * *} P<0.0001\right)$. (B) Spleen-to-body and liver-to-body ratios were lower in mice receiving $\beta$ arr2 $2^{-1-}$ donor cells $\left({ }^{* * *} P<0.0001,1\right.$-way ANOVA). (C) White blood cells (WBCs) were lower over time in mice receiving $\beta$ arr2 $2^{-/-}$cells versus WT or $\beta$ arr ${ }^{-1-}$ cells $\left(^{* * * *} P<0.0001\right.$ for time/row factor, $P<0.01$ for

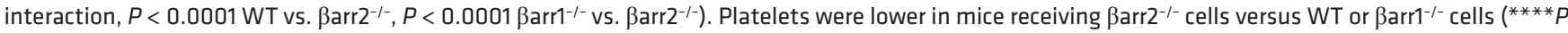
$<0.0001$ for time/row factor, $P<0.05$ for interaction, $P<0.0001$ WT vs. $\beta$ arr2 $2^{-1-}, P<0.0001 \beta$ arr $1^{-1-}$ vs. $\beta$ arr $\left.2^{-1-}\right)$. Hemoglobin was higher over time in mice receiving $\beta$ arr2 $2^{-1-}$ cells versus WT or $\beta$ arr1 ${ }^{-/-}$cells $\left({ }^{* * *} P<0.0001\right.$ for time/row factor, $P<0.0001$ for interaction). Donor chimerism was lower over time in mice receiving $\beta$ arr2 ${ }^{-/-}$cells versus WT or $\beta$ arr1 ${ }^{-/-}$cells $\left(^{* * *} P<0.0001\right.$ for time/row factor, $P<0.05$ for interaction, $P<0.0001$ WT vs. $\beta$ arr2 ${ }^{-/-}$and $\beta$ arr1 ${ }^{-/-}$ vs. $\beta$ arr $2^{-1-}$ ). Two-way ANOVA was used for all analyses. No differences were noted in mice receiving WT vs. $\beta$ arr ${ }^{-1-}$ donor cells for all secondary measures. WT, $n=11 ; \beta \operatorname{arrr}^{-1-}, n=12 ; \beta$ arr2 ${ }^{-1-}, n=16$.

HSC maintenance for success (19). Interestingly, $\beta$-arrestins have been implicated in regulating cell homing (20-24). As described above, mice that received $\beta$ arr $2^{-/-}$donor cells failed to achieve long-term engraftment. To determine if this is due to altered homing of donor cells, we performed in vivo imaging. WT, $\beta a r r 1^{-/-}$, and $\beta a r r 2^{-/-}$KLS cells were harvested and infected with MPLW515L-expressing retrovirus. Prior to injection into recipients, donor cells were labeled with DiR, a fluorescent, lipophilic dye. Microscopy of calvarial bone was utilized to determine the number of donor cells present in marrow 20 hours after transplant (Figure 2, A and B). No differences were noted across the 3 genotypes, indicating proper homing of all cells (Figure 2C). Failure of $\beta$ arr2 ${ }^{-/-}$cells to repopulate marrow is, therefore, not due to altered homing. 


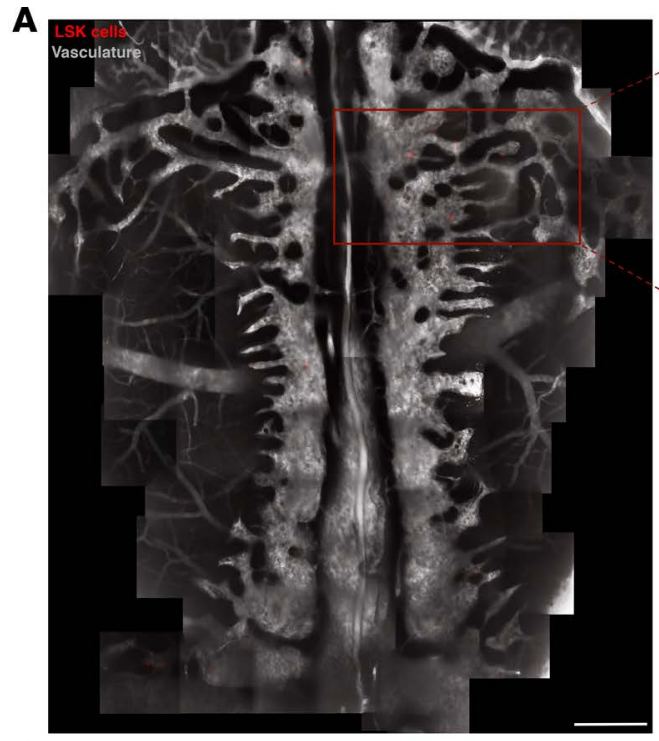

B

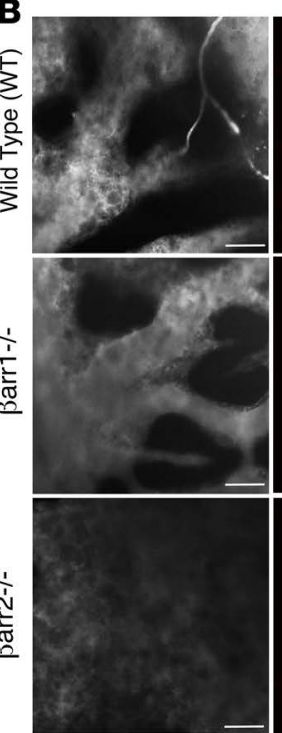

Vasculature (Dex-Cy5)

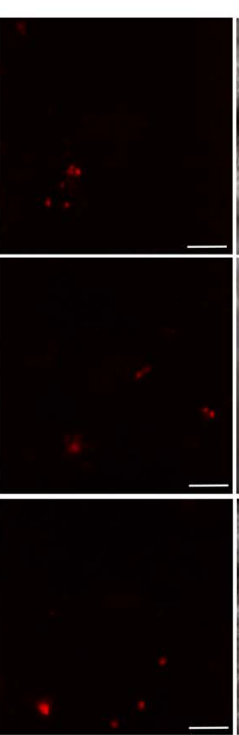

LSK Cells (+ DiR)

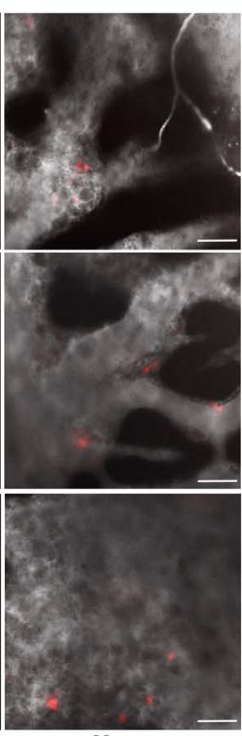

Merge

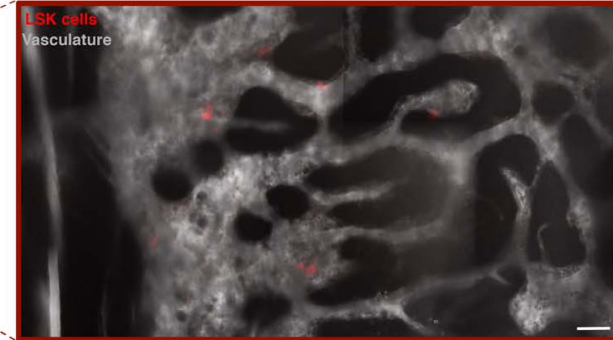

C

Percent Donor Cells

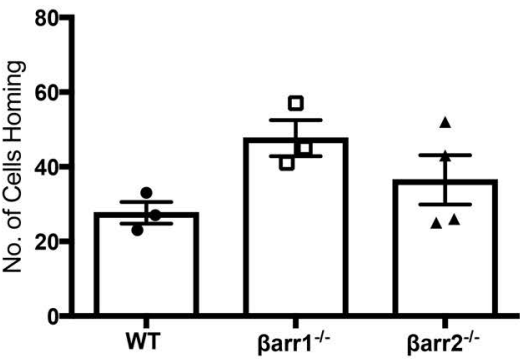

Figure 2. Donor KLS cells from WT, $\beta$-arrestin1-knockout, and $\beta$-arrestin2knockout mice home to marrow. (A) Montage image and zoom of mouse calvarium engrafted with $\mathrm{DiR}^{+}$cells (gray = bone marrow vasculature, Dex-Cy5; red $=\mathrm{DiR}^{+}$cells). Scale bars: calvarium: $400 \mu \mathrm{m}$; zoom image: $75 \mu \mathrm{m}$. (B) Representative images of WT, $\beta$ arr ${ }^{-/-}$, and $\beta$ arr2 ${ }^{-/-}$cells homing to calvarial bone marrow. In vivo imaging performed +20 hours after engraftment. Representative images of $n=3$ experiments. Scale bars: $75 \mu \mathrm{m}$. (C) Cell homing count for WT, $\beta a r r 1^{-1-}$, and $\beta$ arr2 $2^{-1-}$ cells (mean \pm SEM; $n=3$ ). $\beta$ arr1 ${ }^{-1-}, \beta$-arrestin1 knockout; $\beta$ arr2 ${ }^{-1-}$, $\beta$-arrestin2 knockout.

Barr2 ${ }^{-1-}$ KLS cells demonstrate no change in proliferation and both increased apoptosis and decreased self-renewal capabilities in conditions of cellular stress. Our lab and others have shown that lack of $\beta$ arr2 renders various cells more prone to apoptosis $(25,26)$. To investigate mechanisms by which $\beta$ arr $2^{-/-}$cells fail to repopulate marrow, we performed proliferation and apoptosis assays using murine KLS cells. To assess proliferation, we measured 5-ethynyl-2'-deoxyuridine (EdU) incorporation, which detects DNA synthesis in proliferating cells. The percentage of EdU ${ }^{+}$cells was increased similarly in WT, $\beta$ arr $1^{-/-}$, and $\beta$ arr2 ${ }^{-/-}$MPLW515L-treated cells $(72.2 \%, 76.64 \%, 73.93 \%)$ compared with no treatment $(48.59 \%, 44.83 \%, 49.0 \%)$. There was no difference in basal proliferation among the 3 genotypes of cells (Figure $3 \mathrm{~A}$ ). Additionally, $\left[{ }^{3} \mathrm{H}\right]$ thymidine incorporation assays performed measured DNA synthesis in proliferation. Data from both 4- and 24-hour incubation time points were consistent with EdU incorporation (Supplemental Figure 4, A and B). Data from both EdU and $\left[{ }^{3} \mathrm{H}\right]$ thymidine incorporation demonstrate that $\beta$-arrestins do not regulate KLS cell proliferation.

To assess effects of $\beta$-arrestins on apoptosis, annexin $\mathrm{V}$ and propidium iodide were used as markers of apoptosis (27) and dead cells. Interestingly, $\beta$ arr2 ${ }^{-/-}$MPLW515L-expressing KLS cells had a greater than $50 \%$ increase in apoptosis compared with $\beta$ arr $1^{-/-}$MPLW515L-expressing $(P<0.05)$ and WT MPLW515L-expressing KLS cells $(P<0.05)$. There were no significant differences in apoptosis among KLS cells subjected to no treatment and, more importantly, KLS cells treated with MPLWT retrovirus (Figure 3B). These data indicate that KLS cells lacking $\beta$ arr2 are more prone to undergo programmed cell 
A

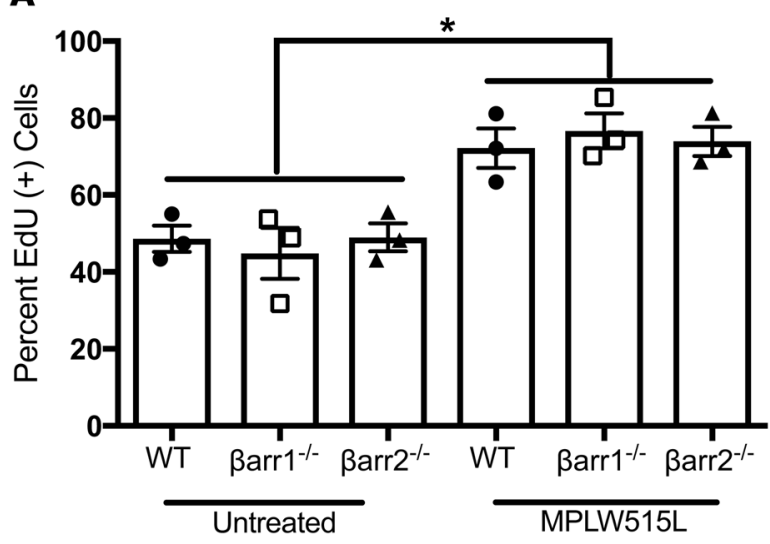

B

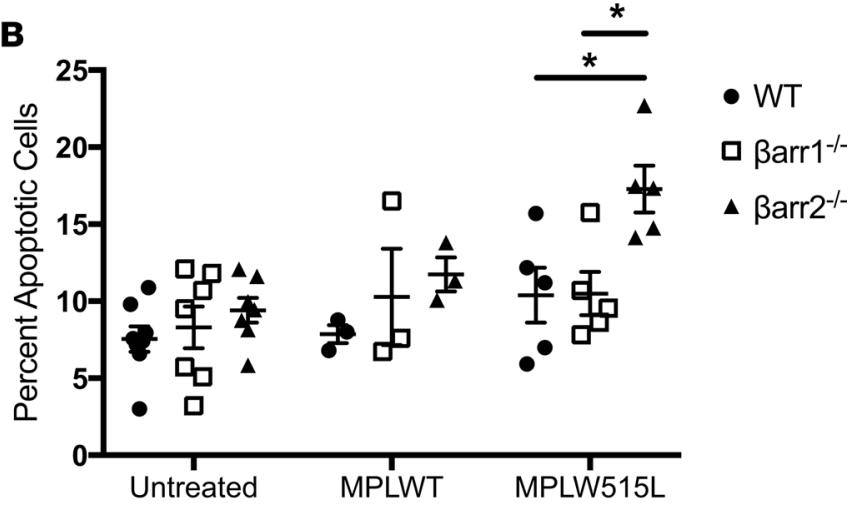

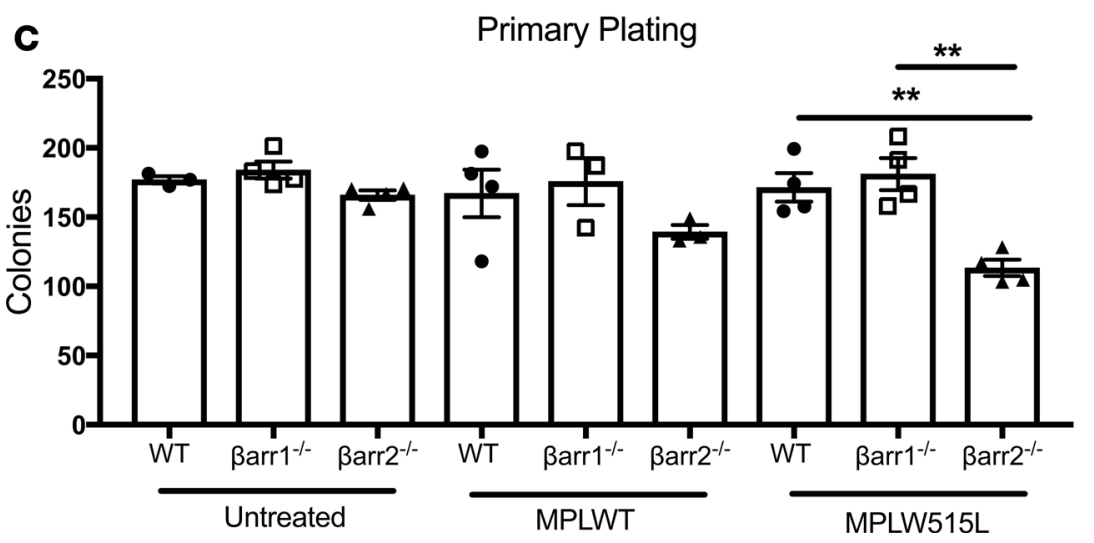

Figure 3. $\beta$-Arrestin2-knockout KLS cells demonstrate no differences in proliferation, increased apoptosis, and decreased self-renewal in the setting of MPLW515L-mutant retrovirus. (A) 5-Ethynyl-2'-deoxyuridine (EdU) incorporation to measure proliferation was increased in each genotype of Should be MPLW515L-treated KLS cells versus untreated control cells of corresponding genotype (*P < 0.05$)$ by 1 -way ANOVA. Within each condition, no differences were observed between genotypes. (B) $\beta$-Arrestin2-knockout ( $\beta$ arr2 ${ }^{-/-}$) MPLW515L-treated cells had significantly increased apoptosis, as measured by annexin staining versus WT MPLW515L-treated and $\beta$-arrestin1-knockout ( $\beta$ arr1 ${ }^{-l^{-}}$) MPLW515L-treated cells by 1 -way ANOVA ( ${ }^{*} P<0.05$ ). (C) Colony formation by KLS cells from WT, $\beta$ arr ${ }^{-/-}$, and $\beta$ arr2 ${ }^{-/-}$mice plated in triplicate in methylcellulose media. $\beta a r r 2^{-/-}$MPLW515L-treated $\mathrm{KLS}^{-1 / l s}$ had decreased colony formation versus WT MPLW515L-treated and $\beta$ arr1 ${ }^{-/-}$MPLW515L-treated cells by 1 -way ANOVA ( ${ }^{*} P<0.01$ ). All experiments were done in triplicate. (A) $n=3$; (B) $n=7$ untreated, $n=3$ MPLWT, $n=5$ MPLW515L; (C) $n>3$.

death under conditions of increased stress, such as those see in our MPLW515L-mutant PMF model, and may explain failure of $\beta a r r 2^{-/-}$cells to repopulate marrow in our mouse model.

Finally, to assess stem cell self-renewal capabilities, we performed colony-forming cell assays with WT, $\beta_{a r r} 1^{-/}$, and $\beta$ arr2 ${ }^{-/-}$KLS cells in no treatment and MPLWT- and MPLW515L-treated conditions. We did not find any differences in number of colonies formed with untreated and MPLWT-treated KLS cells. However, significantly fewer colonies formed with $\beta$ arr2 ${ }^{-1-}$ MPLW515L-treated KLS cells, suggesting that lack of arr $^{-/-}$hinders PMF stem cell self-renewal in vitro (Figure 3C). Taken together, we conclude that PMF development is mediated by $\beta$ arr2, with no phenotypically detectable contribution from $\beta$ arr1. We also conclude that lack of antiapoptotic ßarr2 mediates bone marrow failure of murine HSCs overexpressing MPLW515L.

Barr2 protein expression can be dramatically diminished using a global conditional knockout mouse. To investigate effect of $\beta$ arr2 depletion on PMF progression, we utilized a newly developed global CreER ${ }^{\mathrm{T} 2}-\beta a r r 2$ flox conditional knockout mouse (28). This is an optimal model system, as it allowed us to create mice with PMF under conditions with normal $\beta$ arr2 expression and subsequently observe effects of $\beta$ arr2 depletion. First, we characterized $\beta$ arr2 expression in marrow and spleen, both sites of hematopoiesis consistently affected in PMF. C57BL6/Jßarr2 ${ }^{\mathrm{F} / \mathrm{F}}-\mathrm{CreERT} 2^{+/-}[\mathrm{Cre}(+)]$ and C57BL6/J $\beta$ arr2 ${ }^{\mathrm{F} / \mathrm{F}-\mathrm{CreERT} 2^{-/-}}$[Cre $\left.(-)\right]$ mice were treated with tamoxifen for 5 consecutive days. At various time points after tamoxifen exposure, tissues were harvested and protein lysates were subjected to Western blot analysis. $\beta$ arr1 expression in 

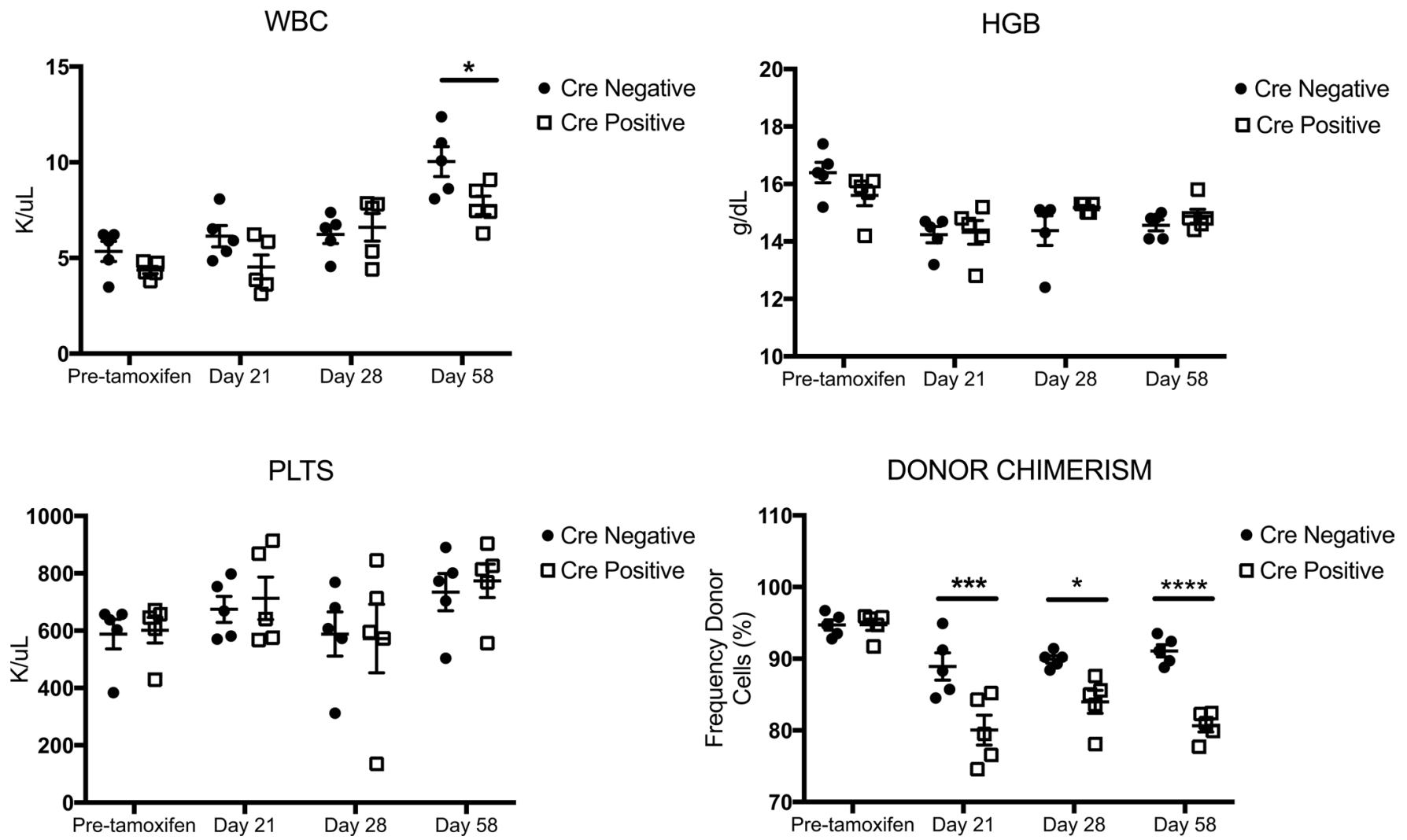

Figure 4. Loss of $\beta$-arrestin2 has effects on KLS cells independent of MPLW515L-mutant retrovirus. KLS cells were harvested from marrow of Cre (+) and Cre (-) donor mice and transplanted in a 1:1 ratio into lethally irradiated WT recipients. Mice were exposed to tamoxifen to induce loss of $\beta$-arrestin2 ( $\beta$ arr2) day 15-19 after transplant after engraftment of donor cells was confirmed. Hematologic parameters and donor chimerism were monitored. There was no change in hematologic parameters over time between Cre (+) and Cre (-) tamoxifen-treated mice, with the exception of a slight increase in WBCs at day 58 after transplant $(P<0.05)$. Cre $(+)$ mice had significantly decreased donor chimerism at day 21,28 , and 58 after transplant versus Cre $(-)$ mice $(P<0.01$ for interaction, ${ }^{* *} P<0.001$ day $21,{ }^{*} P<0.05$ day $28,{ }^{* * *} P<0.0001$ day 58 ) by 2 -way ANOVA. $n=5$ for the entire figure.

spleen was unchanged in tamoxifen-treated $\mathrm{Cre}(+)$ and $\mathrm{Cre}(-)$ mice, whereas $\beta$ arr2 expression was significantly lower in tamoxifen-treated Cre $(+)$ mice over time $(P<0.0001)$ (Supplemental Figure 5A). Findings were consistent in marrow (Supplemental Figure 5B). We observed a $>75 \%$ reduction of $\beta$ arr2 expression by day 10 in spleen and day 8 in marrow. These data demonstrate adequate knockdown of $\beta$ arr2 only in Cre $(+)$ mice and established a timeline for loss of Barr2 expression in this system. Additionally, we obtained complete blood counts for each mouse described above at death to investigate the effect of loss of $\beta$ arr2 on hematopoiesis. Hemoglobin and WBCs were unchanged, although platelets were significantly lower in tamoxifen-treated Cre $(+)$ mice at day 12 after tamoxifen exposure. This difference was transient, as by day +35 after tamoxifen exposure, there were no significant differences in platelet counts in Cre $(+)$ versus Cre (-) tamoxifen-treated mice (Supplemental Figure 6).

Loss of $\beta$ arr2 has an effect on hematopoietic cells independent of MPLW515L-mutant retrovirus. To investigate the effect of loss of $\beta$ arr2 independent of PMF, we harvested donor cells from Cre (+) and Cre (-) donor mice and transplanted these cells in a 1:1 donor-to-recipient ratio into lethally irradiated WT recipient mice. Recipient mice were exposed to tamoxifen day 15-19 after transplant, and hematologic parameters and donor chimerism were monitored. There were no differences in hematologic parameters over time in mice that received Cre $(+)$ versus Cre $(-)$ donor cells, with the exception of a slight increase in WBCs in Cre $(-)$ versus Cre $(+)$ mice at day 58 after transplant $(P<0.05)$. Interestingly, donor chimerism was significantly decreased over time by about $15 \%$ in tamoxifen-treated mice that received $\mathrm{Cre}(+)$ donor cells independent of MPL-mutant retrovirus (Figure 4). This demonstrates that loss of Barr2 has some nonspecific effects on hematopoietic cells.

Mice receiving Cre (+) versus Cre (-) donor cells had significantly different secondary outcomes. To analyze in vivo loss of $\beta$ arr2 after PMF establishment, we utilized our transplant model and $\mathrm{Cre}(+)$ and $\mathrm{Cre}(-)$ donor mice. 
A
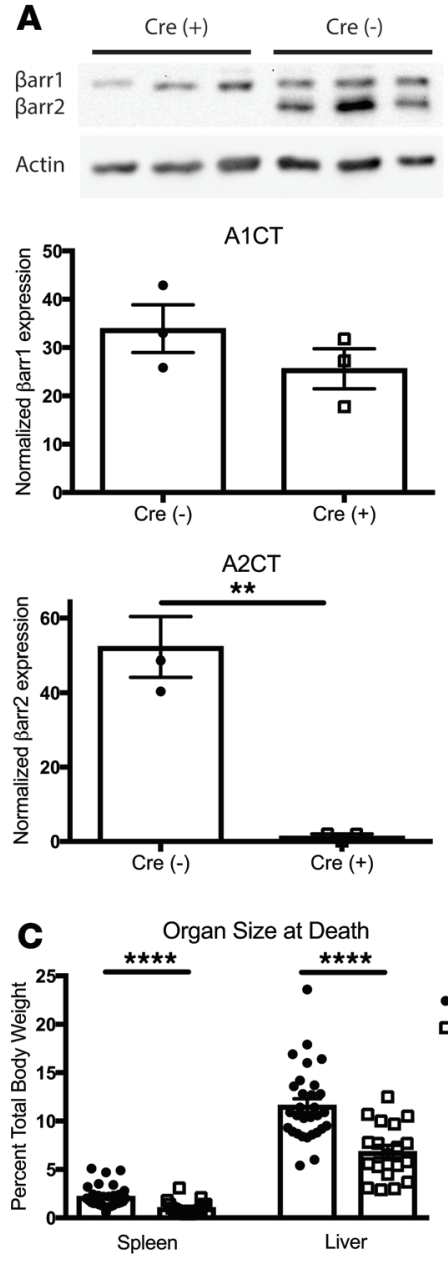

B
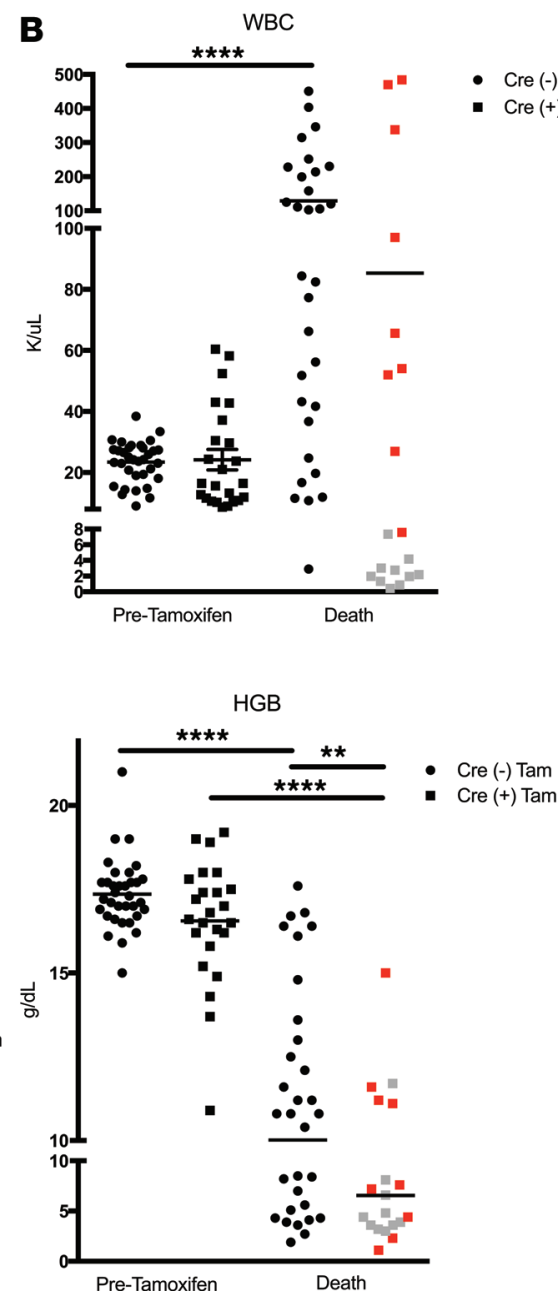

- Cre (-) Tam - Cre (+) Tam
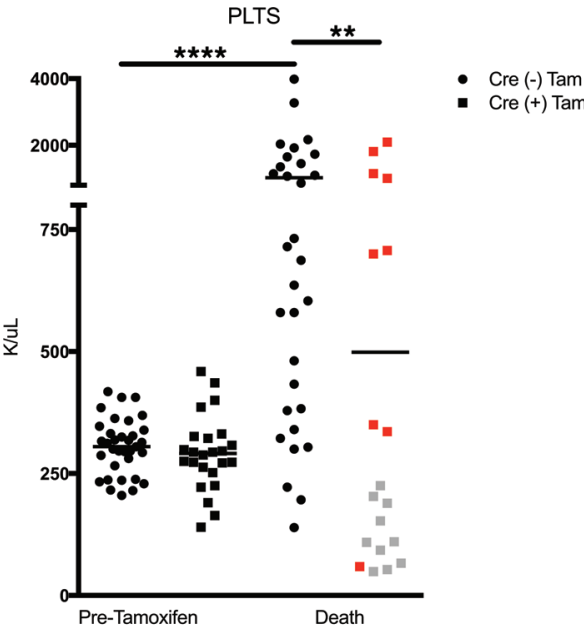

DONOR CHIMERISM

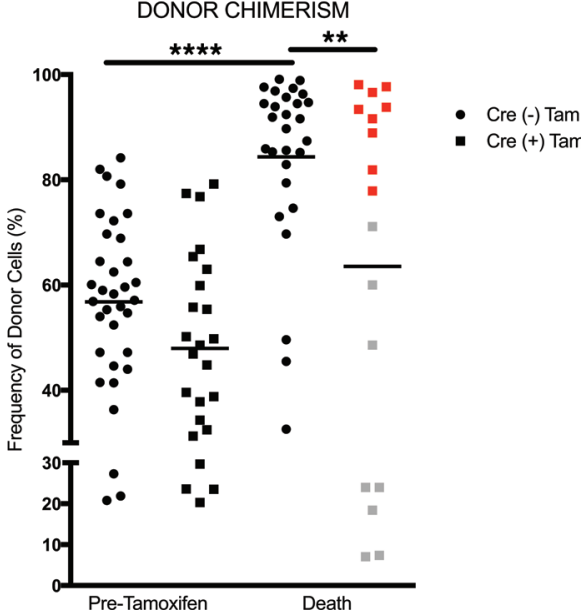

Figure 5. Outcome measures for mice with MPLW515L-mutant primary myelofibrosis receiving $\beta$-arrestin2 conditional knockout donor cells. A retroviral transduction system and cells from Cre (+) and Cre (-) mice were utilized to generate primary myelofibrosis (PMF). Mice received tamoxifen days $15-19$ after transplant. (A) Representative Western blot of $\beta$-arrestin2 ( $\beta$ arr2) protein knockdown in flow-sorted GFP ${ }^{+}$spleen cells 8 days after tamoxifen initiation. $\beta$-Arrestin1 ( $\beta$ arr1) expression was unchanged and $\beta$ arr2 expression was lower in Cre (+) versus Cre (-) cells $\left(1.38 \pm 0.63\right.$ versus $52.3 \pm 8.12,{ }^{* *} P=0.003$, $t$ test) $(n=3)$. (B) White blood cells (WBC) and platelets were higher at death in mice receiving Cre $(-)$ cells versus pretamoxifen. Platelets were also higher at death in mice receiving Cre (-) versus Cre (+) cells. Hemoglobin was lower at death versus pretamoxifen in mice receiving Cre (+) or Cre (-) cells as well as at death in mice receiving Cre (+) versus Cre (-) cells. Donor chimerism was higher at death versus pretamoxifen in mice receiving Cre (-) cells as well as at death in mice receiving Cre (-) versus Cre (+) cells. One-way ANOVA was used for all analyses. ${ }^{* *} P<0.01$; ${ }^{* * *} P<0.0001$. (C) Tamoxifen-treated mice receiving Cre (+) cells had smaller spleen-to-body and liver-to-body ratios (****P<0.0001, unpaired $t$ test) versus Cre (-) controls. Cre (-), $n=34 ;$ Cre $(+), n$ = 24. Red dots indicate mice with PMF. Gray dots represent mice without PMF.

KLS cells were harvested, infected with MSCV-MPLW515L-GFP retrovirus, resorted for GFP positivity, and injected into lethally irradiated WT recipient mice. Presence of PMF was confirmed at day +14 after transplant, at which point mice that received Cre $(+)$ or Cre (-) donor cells had equal ßarr2 expression. Mice were exposed to tamoxifen days 15-19 after transplant and were monitored for survival and secondary endpoints (Supplemental Figure 7). To ensure adequate knockdown of ßarr2 in our model system, we harvested spleens from mice 8 days after tamoxifen initiation, based on results shown in Supplemental Figure 5. Western blot analysis revealed almost complete loss of $\beta$ arr2 expression in transplanted Cre (+) versus Cre $(-)$ donor cells, indicating adequate knockdown of $\beta$ arr2 in vivo (Figure 5A).

We compared outcomes for 4 groups of mice to assess the effect of $\beta$ arr2 depletion on established PMF: (a) Cre (+) donors, tamoxifen treated; (b) Cre (-) donors, tamoxifen treated; (c) Cre (+) donors, vehicle treated; and (d) Cre (-) donors, vehicle treated. Hematologic parameters and donor chimerism were reported at two important time points: prior to tamoxifen exposure (pretamoxifen) and at death. Hematologic parameters and donor chimerism were similar between mice receiving Cre $(+)$ or $\mathrm{Cre}(-)$ cells before tamoxifen. WBCs were 

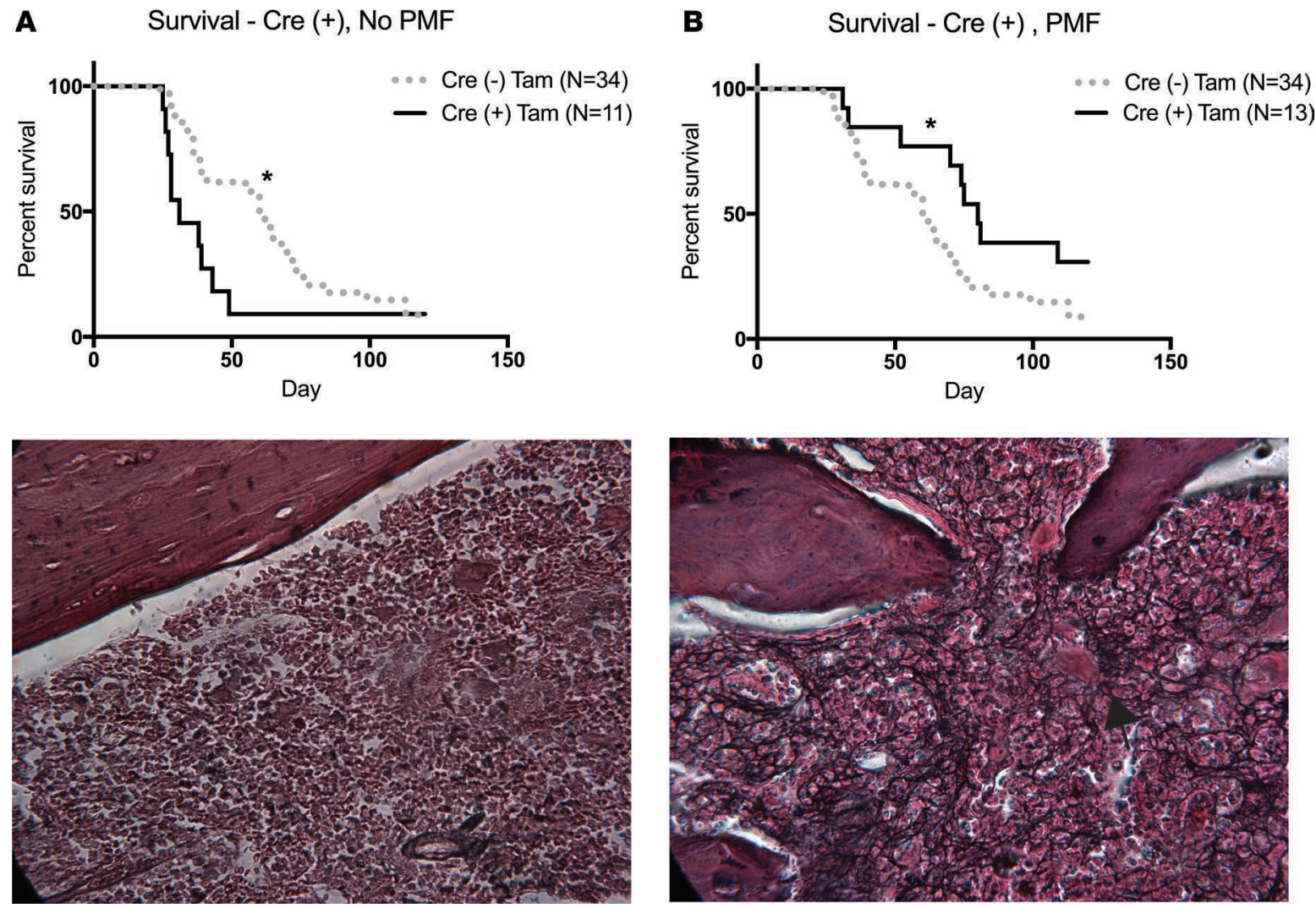

Figure 6. Survival is improved in tamoxifen-treated conditional $\beta$-arrestin2-knockout mice with primary myelofibrosis. Tamoxifen-treated mice that received Cre (+) donor cells separated into two groups: (a) primary myelofibrosis (PMF) and (b) no PMF. (A) Median survival in mice without PMF was 31 days compared with 60 days for tamoxifen-treated mice that received Cre $(-)$ cells by log-rank Mantel-Cox testing $\left({ }^{*} P=0.03\right)$. Representative image (original magnification, $\times 40)$ of reticulin-stained femur from a mouse without PMF. There is minimal fibrosis. (B) Median survival in mice with PMF was longer at 80 days compared with 60 days for tamoxifen-treated mice that received Cre (-) cells by log-rank Mantel-Cox testing ( $\left.{ }^{*} P=0.04\right)$. Representative image (original magnification, $\times 40$ ) of reticulin-stained femur from a mouse with PMF. Marked fibrosis (arrow) is present.

significantly higher at death versus pretamoxifen in tamoxifen-treated mice that received Cre $(-)$ cells (129 K/ $\mu 1$ versus $23.4 \mathrm{~K} / \mu 1, P<0.0001)$. In contrast, WBCs at death versus pretamoxifen were the same for mice that received Cre $(+)$ cells. Similarly, platelets were higher at death in mice receiving Cre $(-)$ cells versus pretamoxifen $(1,026 \mathrm{~K} / \mu \mathrm{l}$ versus $305.4 \mathrm{~K} / \mu 1, P<0.0001)$, with no difference noted in mice that received Cre $(+)$ cells at the same time points. Platelets were lower at death in tamoxifen-treated mice that received Cre $(+)$ versus Cre $(-)$ cells $(P<0.01)$. Hemoglobin was lower at death in mice that received Cre $(+)$ donor cells versus pretamoxifen $(P<0.0001)$ and versus mice at death that had received Cre $(-)$ cells $(P<0.01)$. Interestingly, donor chimerism was higher at death in mice receiving Cre $(-)$ cells versus pretamoxifen $(P<0.0001)$ as well as at death in mice receiving Cre $(-)$ versus Cre $(+)$ cells $(P<0.01)$ (Figure 5B). Spleen-to-body $(P<0.001)$ and liverto-body $(P<0.001)$ ratios were notably smaller in tamoxifen-treated mice that received Cre $(+)$ cells (Figure $5 \mathrm{C})$. Collectively, these data indicate significant differences in outcome measures between tamoxifen-treated mice receiving Cre $(+)$ versus Cre $(-)$ donor cells. Interestingly, mice that received Cre $(-)$ cells had more dramatic hematologic abnormalities compared with mice that received Cre $(+)$ cells. When we examined survival among all mice, however, no differences were noted (Supplemental Figure 8).

Tamoxifen-treated mice that received Cre $(+)$ cells had two disparate clinical courses. In experiments to establish conditional knockout of $\beta$ arr2, we observed two disparate clinical courses among tamoxifen-treated mice that received Cre (+) cells: (a) PMF and (b) no PMF. This disparate course was consistently observed within our experimental animals and is depicted with red (PMF) versus gray (no PMF) dots in Figure 5B. Median survival for tamoxifen-treated mice that received Cre $(+)$ cells without PMF was 31 days versus 60 days for tamoxifen-treated mice that received Cre $(-)$ cells by log-rank Mantel-Cox testing (hazard ratio $0.48,95 \% \mathrm{CI}$ $0.15-0.88, P=0.03)$. On histology, no mice had significant fibrosis by reticulin stain of marrow and spleen, consistent with lack of PMF (Figure 6A). Alternatively, median survival for tamoxifen-treated mice that 


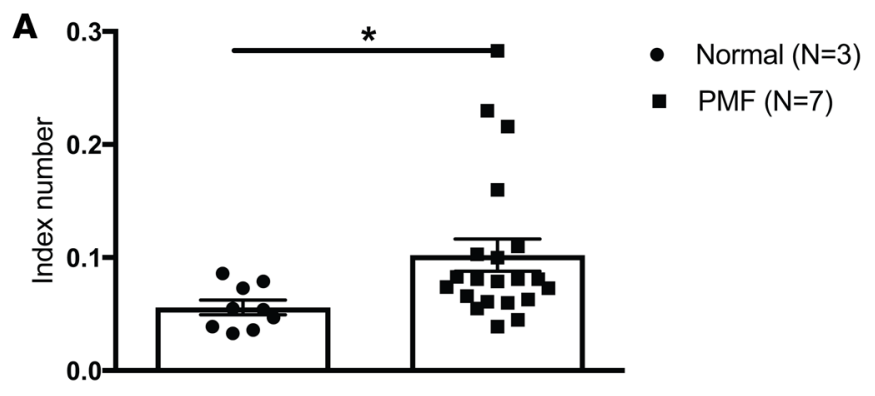

B
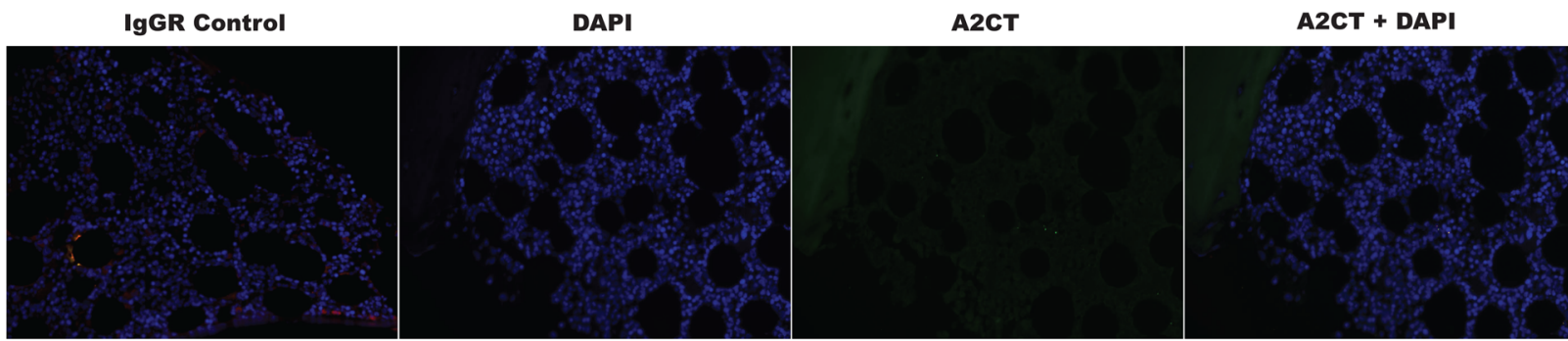

PMF
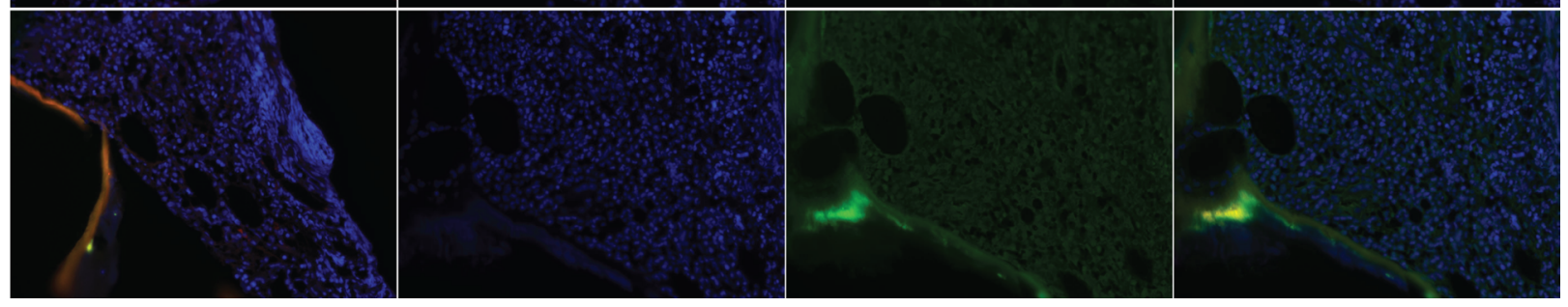

Figure 7. Human primary myelofibrosis samples have increased $\beta$-arrestin2 expression versus normal marrow controls. Primary human bone marrow samples from patients with primary myelofibrosis (PMF) and normal bone marrow controls were stained for $\beta$-arrestin2 ( $\beta$ arr2) utilizing A2CT antibody. A second slide per sample was stained using nonimmune lgG control, and immunofluorescence was quantified. $\beta$ arr2 expression was normalized to IgG background and DAPI staining. (A) Primary human PMF samples had increased $\beta$ arr2 expression versus normal marrow controls ( ${ }^{*} P=0.048$ by $t$ test). All images were quantified in triplicate. (B) Representative images of normal human bone marrow and PMF samples. Original magnification, $\times 20$.

received Cre $(+)$ cells with PMF was significantly longer at 80 days versus 60 days in tamoxifen-treated mice that received Cre (-) cells by log-rank Mantel-Cox testing (hazard ratio 2.07, 95\% CI 1.03-3.72, $P=0.04$ ). All mice displayed marked fibrosis by reticulin stain consistent with PMF (Figure 6B). We also observed different secondary outcomes between these two groups. Tamoxifen-treated Cre $(+)$ mice without PMF had lower WBCs, platelets, spleen and liver size, and donor chimerism at death versus Cre (+) mice with PMF (Supplemental Figure 9, A and B). Upon analysis of data described above, we determined that mice without PMF suffered loss of donor cells with resultant marrow aplasia. Given that these animals did not have PMF, we excluded them and analyzed only tamoxifen-treated mice receiving Cre $(+)$ and Cre $(-)$ cells with PMF.

When we analyzed secondary outcomes in tamoxifen-treated mice that received Cre $(+)$ and Cre $(-)$ cells and had PMF, we observed several differences between measurements before tamoxifen treatment versus at death within each genotype of cells. However, there were no significant differences at death in mice that received Cre (+) versus Cre (-) cells (Supplemental Figure 10, A and B). These results indicate that tamoxifen-treated mice that received Cre $(+)$ cells with PMF have an attenuated disease course with improved survival versus controls, although they experienced no alternations in secondary outcomes measures.

To assess in vivo apoptosis, we harvested KLS cells from Cre $(+)$ and Cre $(-)$ donor mice, infected them with MSCV-MPLW515L-GFP retrovirus, resorted for GFP positivity, and injected them into lethally irradiated WT recipient mice. Mice were treated with tamoxifen 15-19 days after transplant, and mice were euthanized on day 5 after initiation of tamoxifen based on the results shown in Supplemental Figure 5. Murine femurs were harvested, embedded in paraffin, and subjected to TUNEL staining. Tamoxifen-treated mice that received Cre (+) donor cells had more $\mathrm{TUNEL}^{+}$cells in comparison to tamoxifen-treated mice that received Cre (-) donor cells, indicating increased apoptosis with tamoxifen exposure and loss of $\beta$ arr2 in hematopoietic cells (Supplemental Figure 11). This is consistent with our in vitro data. 
Barr2 expression is increased in human PMF. To determine if $\beta$ arr2 expression is altered in human PMF, we examined marrow samples from patients with PMF versus normal marrow controls. Paraffin-embedded samples were stained with $\beta$ arr2 primary antibody and expression was calculated and normalized by IgG and Hoechst staining. Notably, PMF samples had almost a 2-fold increase in $\beta$ arr2 expression versus controls $(P<0.05)$ (Figure 7).

\section{Discussion}

$\mathrm{PMF}$ is associated with debilitating constitutional symptoms and significant risk of transformation to acute leukemia. It is associated with driver mutations, including JAK2, calreticulin, and MPL. Therapies targeting the JAK/STAT pathway are effective, although not curative, in patients both with and without JAK2 mutations $(29,30)$. This indicates that other pathways are involved in disease pathogenesis and could be exploited for novel therapies. Fereshteh et al. first investigated the relationship between CML and $\beta$ arr2 (12). Given that CML and PMF belong to the same family of diseases, we investigated the relationship between $\beta$-arrestins and PMF.

Mammalian cells express 4 subtypes of arrestins, with $\beta$ arr1 and $\beta$ arr2 being expressed ubiquitously throughout the body. Different cells have varying levels of $\beta$-arrestin expression and, accordingly, $\beta$ arr 1 and $\beta$ arr2 serve different functions in different cells and disease-specific states. $\beta$ arr2, specifically, has been implicated in the pathogenesis of hepatocellular carcinoma and breast and prostate cancer (31-34). In contrast to data from Fereshteh et al., Qin et al. demonstrated that $\beta$ arr1 mediates CML progression due to effects on histone $\mathrm{H} 4$ acetylation (35). Acute lymphoblastic leukemia (ALL) is another hematologic malignancy characterized by excess proliferation of immature lymphoid cells. Whereas Fereshteh et al. demonstrated decreased HSC self-renewal in $\beta$ arr2-deficient CML cells, Shu et al. demonstrated that $\beta$ arr1 regulates leukemia HSC self-renewal in ALL (36). These opposing findings are likely secondary to functional redundancy between arrestins. In our experiments, we show that $\beta$ arr2 expression is increased in human PMF versus controls, suggesting potential involvement of $\beta$ arr 2 in disease pathogenesis. Using $\beta a r r 1^{-/-}$and $\beta$ arr2 $2^{-/}$mice, we also show that $\beta$ arr2 is involved in the pathogenesis of PMF with no phenotypic involvement of $\beta$ arr1.

Interestingly, as early as day 14 after transplant in our global knockout PMF mouse model, mice that received $\beta$ arr $2^{-/-}$cells had minimal donor chimerism, suggesting that altered long-term self-renewal is not the only mechanism of $\beta$ arr2 involvement in PMF. In order to repopulate marrow, donor cells must home to marrow, engraft, proliferate, and self-renew. As stated above, several publications have shown that arrestins are implicated in chemotaxis and migration in various cell types (37-39). Through in vivo microscopy experiments, we demonstrated equitable homing of $\beta$ arr $2^{-/-}$versus WT and $\beta$ arr $1^{-/}$cells, indicating that $\beta$ arr2 is not required for proper homing of cells to marrow. $\left[{ }^{3} \mathrm{H}\right]$ thymidine and EdU incorporation assays to assess proliferation showed no differences in $\beta$ arr $2^{-/-}$versus control cells, demonstrating that $\beta$ arr2 does not affect proliferation in physiologic and stressed conditions. Our lab has previously shown that $\beta$ arr2 deficiency renders cells prone to apoptosis (26). Interestingly, $\beta$ arr2 ${ }^{-/-}$KLS cells had increased apoptosis when subjected to treatment with MPLW515L-mutant retrovirus, thus providing a mechanism for failed short-term engraftment of donor cells specifically in our disease model.

Although a defect in self-renewal capabilities of $\beta$ arr $2^{-/-}$KLS cells may certainly be contributing to failed engraftment, it is known that transplants utilizing $\beta$ arr $2^{-/-}$donor cells are successful. In a paper by Hollingsworth et al., for example, the authors transplanted $\beta$ arr $2^{-/-}$donor cells into WT recipients and WT cells into $\beta$ arr $2^{--}$mice. In both scenarios, high and equal engraftment of donor cells was achieved in recipient mice, demonstrating that it is possible to achieve long-term engraftment of $\beta$ arr $2^{-/}$cells in a murine transplant model (40). However, these transplants were done with unmanipulated cells, as opposed to the cells used in our PMF model that are characterized by excessive cell proliferation and associated cellular stress. This again supports our conclusion that additional mechanisms, i.e., increased apoptosis with lack of antiapoptotic $\beta$ arr2, are responsible for the lack of engraftment of $\beta$ arr $2^{-/-}$KLS cells, specifically in a MPLW515L-mutant model of disease.

Tools to regulate $\beta$-arrestin function in a time-dependent or controlled manner in vivo (small-molecule inhibitors, single-chain variable fragments, etc.) are lacking, making it difficult to assess the full potential of $\beta$-arrestins as therapeutic targets. Kotula et al. have effectively applied an aptamer chimera targeting $\beta$ arr2 to $\mathrm{CML}$ cell lines and primary human samples, demonstrating that it is possible to effectively inhibit $\beta$ arr2 in vitro (41). In this paper, our mouse model generated preliminary data supporting $\beta$ arr2 as a therapeutic 
target in PMF and may provide impetus to invest efforts to develop tool compounds and ultimately therapeutic agents targeting $\beta$ arr2. Perhaps more importantly, these tools and findings may be more broadly applicable to other malignancies.

In our conditional knockout model, all mice were phenotypically identical prior to tamoxifen exposure. Interestingly, in tamoxifen-treated mice that received Cre $(+)$ donor cells, we observed two distinct clinical outcomes and identified a subgroup of tamoxifen-treated animals that did not have PMF. We hypothesize that variable knockdown of $\beta$ arr2 across mice accounts for the divergent outcomes. Mice without PMF had more profound loss of $\beta$ arr2, resulting in increased apoptosis with acute loss of donor cells and marrow failure. Cre (+) mice with PMF had increased donor chimerism over time compared with baseline, with significantly elevated WBCs, platelets, and hepatosplenomegaly at death. Although tamoxifen-treated mice that received Cre (+) cells had no significant differences in outcome measures at death compared with Cre $(-)$ controls, these mice demonstrated improved survival. We hypothesize that some loss of $\beta$ arr2, with resultant apoptosis of malignant cells, led to this notable survival advantage.

Based on our data demonstrating a $10 \%-15 \%$ loss of donor chimerism with tamoxifen treatment of mice that received Cre $(+)$ donor cells independent of MPLW515L-mutant retrovirus, we can conclude that some loss of chimerism seen in our conditional knockout PMF mouse model is secondary to the nonspecific effect of acute loss of $\beta$ arr2. Loss of donor chimerism is much more pronounced in the setting of MPLW515L-mutant retrovirus, thus supporting our conclusion that there are some disease-specific effects of loss of $\beta$ arr2.

Our data establishes a relationship between $\beta$ arr2 and PMF, indicates that $\beta$ arr2 alters progression of disease, and demonstrates that antiapoptotic ßarr2 mediates bone marrow failure of murine HSCs overexpressing MPLW515L. In many cancers, malignant cell growth is uninhibited, due to the ability of these cells to evade apoptosis. Therefore, $\beta$-arrestin and its' involvement in regulating apoptosis is an attractive target in cancer. Additional studies both in mouse and in human PMF are warranted. Ultimately, $\beta$ arr2 may serve as a therapeutic target in this morbid disease for which effective therapies are limited.

\section{Methods}

Mice. Mice were males, aged 8-12 weeks. WT (The Jackson Laboratory), $\beta$ arr $1^{-/-}$(generated in-house), and $\beta$ arr2 ${ }^{-/-}$(generated in-house) mice were on a C57BL/6J (CD45.2) background, and recipients were

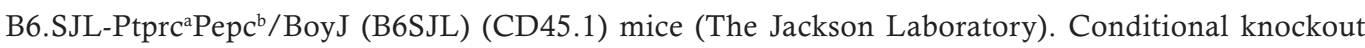
mice, C57BL6/Jßarr2 ${ }^{\mathrm{F} / \mathrm{F}}-\mathrm{CreERT2} 2^{+/-}[\mathrm{Cre}(+)]$ and C57BL6/J $\beta$ arr2 ${ }^{\mathrm{F} / \mathrm{F}}-\mathrm{CreERT}^{-/-}[\mathrm{Cre}(-)]$, were generated and littermate controls were utilized (28). Animals were maintained on antibiotic water for 2 weeks after transplant.

KLS (HSC) isolation and PMF generation for KLS transplants. Marrow cells were harvested from donor mouse femurs $(42,43)$. c-Kit ${ }^{+}$cells were isolated using MACS microbeads and LS columns according to the manufacturer's specifications (Miltenyi). C-kit ${ }^{+}$cells were incubated with antibodies to c-Kit, Sca-1, CD3, CD4, CD8a, CD11b, B220, Ter119/erythroid cells, and Gr-1 (Biolegend) and were sorted for KLS markers (c-Kit $\left.{ }^{+}, \mathrm{Sca}^{+}{ }^{+}, \mathrm{Lin}^{-}\right)$by flow cytometry (FACSDiva, BD Biosciences). Cells were cultured with X-Vivo media (Lonza) supplemented with 10\% FBS, 1\% penicillin/streptomycin, $100 \mathrm{ng} / \mathrm{ml}$ stem cell factor, and $20 \mathrm{ng} / \mathrm{ml}$ thrombopoietin (R\&D Systems). Cells were infected with MSCV-hMPLW515L-GFP and resorted for GFP positivity. Fifty thousand $\mathrm{GFP}^{+}$cells and $8 \times 10^{5}$ support cells from recipients were injected retro-orbitally into lethally irradiated (900 cGy) congenic WT recipient mice. Mice were monitored daily for weight and signs of morbidity. Premorbid animals were euthanized and relevant data were collected. Blood was drawn weekly after transplantation. Complete blood counts were measured using a Hemavet counter (Drew Scientific), and number of recipient-origin (CD45.1) versus donor-derived cells (CD45.2) or donor chimerism was analyzed by flow cytometry using BD FACSCanto and FlowJo software (Treestar).

Whole marrow transplants. Marrow cells were harvested from donor WT, $\beta$ arr $1^{-/-}$, and $\beta a r r 2^{-/-}$mouse femurs and red blood cells (RBCs) were lysed using RBC lysis buffer (eBiosciences). Cells were subsequently injected retro-orbitally into lethally irradiated (900 cGy) congenic WT recipient mice in a 1:1 donor-to-recipient ratio. Mice were monitored for weight and signs of morbidity. Secondary outcome measures, including complete blood counts and donor chimerism, were followed over time.

Study design and conditional knockout transplants. KLS cells were collected from Cre $(+)$ or Cre (-) mice and were transplanted as described above. PMF presence was determined +14 days after transplant. Mice with WBCs of $>8 \times 10^{3} / \mathrm{ml}$ and $>25 \%$ donor-derived cells (CD 45.2) were included. In preliminary experiments, 
95\% of mice meeting these criteria developed PMF (data not shown). Mice received tamoxifen $75 \mathrm{mg} / \mathrm{kg}$ or corn oil vehicle (MilliporeSigma) by intraperitoneal injection day $+15-19$ after transplant. Animals were monitored for survival and secondary endpoints. Only male mice were utilized to ensure no confounding hormonal or immune effects of tamoxifen in females (44-46).

Proliferation assays. For both assays, KLS cells were harvested from WT, $\beta$ arr1 $1^{-/}$, and $\beta$ arr2 $2^{-/-}$mice. DNA synthesis was measured using $\left[{ }^{3} \mathrm{H}\right]$ thymidine incorporation. Twenty-four and four hours prior to assay performance, $\left[{ }^{3} \mathrm{H}\right]$ thymidine $(0.5 \mu \mathrm{Ci} / \mathrm{ml})$ was added to medium of each well $(25,000 /$ well $)$. Cells were washed with PBS, and incorporation of $\left[{ }^{3} \mathrm{H}\right]$ thymidine into DNA strands was determined using a Tri-Carb 2800 liquid scintillation counter (Perkin-Elmer) and Lefko Fluor scintillation cocktail (RPI). To measure active DNA synthesis, cells were stained per manufacturer's instructions with EdU (47) (Life Technologies). EdU ${ }^{+}$cells were measured by flow cytometry. All experiments were performed in triplicate.

Apoptosis assays. KLS cells from WT, $\beta$ arr $1^{-/}$, and $\beta a r r 2^{-1-}$ mice were harvested; cultured with MPLW515L-mutant retrovirus versus control; and were subjected to PacBlue annexin V (Biolegend) and propidium iodide (Thermo Fisher) staining. The percentage of positive cells was determined utilizing flow cytometry. Experiments were performed in triplicate. Apoptosis was also assessed by TUNEL assay (R\&D Systems). Paraffin-embedded murine femurs were stained per the manufacturer's instructions. Hoechst (1:500) (Invitrogen) was also applied to measure nucleus expression. Immunofluorescence was measured utilizing a Zeiss LSM510 laser-scanning microscope.

Colony-forming cell assays. KLS cells were harvested from marrow of WT, $\beta$ arr $1^{-/-}$, and $\beta$ arr $2^{-/-}$mice and were cultured with MPLW515L-mutant retrovirus versus control (MPLWT and no treatment). Ten thousand cells were plated with complete methylcellulose medium (Stem Cell Technologies), and colonies were counted 8-10 days after plating. Experiments were performed in triplicate.

Immunoblotting. Immunoblotting for $\beta$ arr1 and $\beta$ arr2 expression was performed (48). To assess extent and time required for induced loss of $\beta$ arr2 expression, Cre $(+)$ global $\beta$ arr2 conditional knockout mice were treated with tamoxifen and tissues were harvested at various time points. Lysates were prepared from spleen and marrow. Cells were lysed in glycerol lysis buffer containing protease and phosphatase inhibitors (Pierce BioTechnology). Lysates were rotated for 1 hour at 4 degrees to solubilize lysate, and insoluble material was separated by centrifugation. Protein concentration was measured using a BCA Protein Assay Kit (Thermo Fisher), and $20 \mu \mathrm{g}$ of protein was loaded onto $10 \%$ Tris-glycine polyacrylamide gels (Life Technologies) with subsequent transfer to nitrocellulose membranes. $\beta$ arr1 was detected using rabbit polyclonal A1CT antibody (1:2,000). $\beta$ arr2 was detected using rabbit polyclonal A2CT antibody $(1: 1,000)$ (49). Secondary antibody with chemiluminescent detection was performed using SuperSignal West Pico Reagent (Thermo Fisher). Immunoblots were visualized and quantified using Bio Imaging System (Syngene). Levels of arrestin expression were normalized to $\beta$-actin $(1: 5,000)$ (MilliporeSigma).

KLS cell labeling. WT, $\beta$ arr1 $1^{-/}$, and $\beta a r r 2^{-/-}$KLS cells were isolated and labeled with lipophilic DiR fluorescent dye (Invitrogen). Cells were resuspended at a density of $2.5 \times 10^{6}$ cells $/ \mathrm{ml}$ in complete culture media (RPMI-1640 + 10\% FBS) and incubated with $25 \mu \mathrm{M}$ DiR for 30 minutes at $37^{\circ} \mathrm{C}$. After incubation, cells were washed in PBS, counted, and resuspended at desired concentrations for mouse engraftments as above (50). Dye labeling efficiency was tested via flow cytometry analysis using a FACSCanto II cytometer (BD Biosciences).

In vivo cell homing. B6.SJL-Ptprc ${ }^{\mathrm{a}} \mathrm{Pepc}^{\mathrm{b}} /$ BoyJ mice were engrafted with fluorescently labeled WT, $\beta a r r 1^{-/-}$, and $\beta$ arr2 ${ }^{-/-}$KLS cells and were prepared for in vivo imaging 20 hours after engraftment. Mice were anesthetized using isoflurane throughout the procedure and a rectangular scalp incision was made, revealing intact underlying cortical bone. Fluorescently labeled high-molecular-weight dextran (Dex-Cy5) was administered via tail vein to facilitate vasculature imaging. Mice were placed in a specially designed restrictor, and a cover slip covered exposed calvarial bone. High-resolution images were obtained through intact skull using a Leica SP5 confocal and multiphoton microscope with a $\times 20 / 0.40$ NA objective lens. DiR and Dex-Cy5 imaging utilized a femtosecond Titanium:sapphire laser (Chameleon) and an Argon cs laser. Images were captured using Leica LAS-AF software using line and frame averaging. Calvarial marrow was subdivided into numbered anatomical areas, and overlapping $\times 20$ images were captured of the entire region. After the procedure, images were merged to generate a montage, allowing total cell homing counts for each cell type to be determined.

Immunohistochemistry. Femurs were fixed in $4 \%$ paraformaldehyde, decalcified, and embedded in paraffin. Cryostat sections were stained for H\&E and for reticulin, and images were obtained using a Leica DM6 B light/fluorescence microscope. 
Human sample imaging. Human marrow samples were obtained from the University of North Carolina School of Medicine Department of Pathology. One slide per sample was stained using A2CT (1:500) and Hoechst to measure nucleus expression (1:500) (Invitrogen). One slide per sample was stained using nonimmune mouse IgG and rabbit IgG (Santa Cruz). Immunofluorescence was measured utilizing a Zeiss LSM510 laser-scanning microscope. $\beta$ arr2 and nucleus expression were quantified using Imaris $8.1 \mathrm{com}$ puter software. $\beta$ arr2 expression was normalized to IgG control and Hoechst expression. Index number was calculated using the following: (A2CT signal from sample - A2CT signal from IgG control)/DAPI.

Statistics. Data were analyzed using standard tests, including 2-tailed Student's $t$ test and 1-way and 2-way ANOVA. All ANOVA analyses were done with post-test Bonferroni multiple comparisons. To detect $30 \%-40 \%$ difference in survival with $80 \%$ power at an $\alpha$ of 0.05 and with a hazard ratio of at least 2 using a 1-sided test, we estimated that 10 animals would be required per group per experiment. Sample size was monitored to ensure that excess studies were not performed. Kaplan-Meier curves with log-rank tests were used to analyze survival. Error bars represent mean \pm SEM. $P$ values of less than 0.05 were considered significant.

Study approval. All animal studies were performed in accordance with recommendations in the Guide for the Care and Use of Laboratory Animals (National Academies Press, 2011) from the NIH. The work was approved by the Duke IACUC (protocol A014-17-01). The protocol was also approved by the Institutional Biosafety and Recombinant DNA Committee (protocol 11-0033-0) at Duke University. Animals were bred and maintained in IACUC-approved facilities.

\section{Author contributions}

LAMR, JWW, JK, BT, LH, TP, HY, and MC performed experiments; LAMR, JWW, and JK designed the research; LAMR analyzed data and wrote the manuscript; YF provided samples and assisted with human samples research design; DS assisted with designing in vivo microscopy experiments; WC, JKLW, RTP, and RJL designed experiments and interpreted results; and RJL reviewed the manuscript and supervised the research project.

\section{Acknowledgments}

We thank R. Levine for providing the MPLW515L-mutant PMF viral constructs; D. Addison and Q. Lennon for administrative support; M. Cook for cell sorting; J. Snyder for assistance with quantification of human samples images; C. Ingersoll for animal breeding; L. Wingler for critical review of the manuscript; and N. Chao, S. Sarantopoulos and P. Doan for scientific discussion. This work was supported by NIH grants R01-HL016037-43 to RJL and T32 HL007057-38 to LAMR. RJL is an investigator with the Howard Hughes Medical Institute. LAMR received support from the American Society of Hematology and the Duke Medicine Chair's Research Award.

Address correspondence to: Robert J. Lefkowitz, Duke University Medical Center Box 3821, Durham, North Carolina 27710, USA. Phone: 919.684.2974; Email: 1efko001@receptor-biol.duke.edu.

1. Tefferi A. Polycythemia vera and essential thrombocythemia: 2012 update on diagnosis, risk stratification, and management Am J Hematol. 2012;87 (3):285-293.

2. Tefferi A. Essential thrombocythemia, polycythemia vera, and myelofibrosis: current management and the prospect of targeted therapy. Am J Hematol. 2008;83 (6):491-497.

3. Vannucchi AM, et al. Philadelphia chromosome-negative chronic myeloproliferative neoplasms: ESMO Clinical Practice Guidelines for diagnosis, treatment and follow-up. Ann Oncol. 2015;26 Suppl 5:v85-v99.

4. Tefferi A. Primary myelofibrosis: 2017 update on diagnosis, risk-stratification, and management. Am J Hematol. 2016;91 (12):1262-1271.

5. Deeg HJ, et al. Hematopoietic cell transplantation as curative therapy for patients with myelofibrosis: long-term success in all age groups. Biol Blood Marrow Transplant. 2015;21 (11):1883-1887.

6. Kralovics R, et al. A gain-of-function mutation of JAK2 in myeloproliferative disorders. N Engl J Med. $2005 ; 352$ (17):1779-1790.

7. Beer PA, et al. MPL mutations in myeloproliferative disorders: analysis of the PT-1 cohort. Blood. 2008;112 (1):141-149.

8. Baxter EJ, et al. Acquired mutation of the tyrosine kinase JAK2 in human myeloproliferative disorders. Lancet. 2005;365 (9464):1054-1061.

9. Delhommeau F, Jeziorowska D, Marzac C, Casadevall N. Molecular aspects of myeloproliferative neoplasms. Int J Hematol. 2010;91 (2):165-173.

10. James C. The JAK2V617F mutation in polycythemia vera and other myeloproliferative disorders: one mutation for three diseases? Hematology Am Soc Hematol Educ Program. 2008:69-75. 
11. Jatiani SS, Baker SJ, Silverman LR, Reddy EP. Jak/STAT pathways in cytokine signaling and myeloproliferative disorders: approaches for targeted therapies. Genes Cancer. 2010;1 (10):979-993.

12. Fereshteh M, et al. $\beta$-Arrestin2 mediates the initiation and progression of myeloid leukemia. Proc Natl Acad Sci USA. 2012;109 (31):12532-12537.

13. Shenoy SK, Lefkowitz RJ. $\beta$-Arrestin-mediated receptor trafficking and signal transduction. Trends Pharmacol Sci. 2011;32 (9):521-533.

14. Kovacs JJ, Hara MR, Davenport CL, Kim J, Lefkowitz RJ. Arrestin development: emerging roles for beta-arrestins in developmental signaling pathways. Dev Cell. 2009;17 (4):443-458.

15. Zhang P, He X, Tan J, Zhou X, Zou L. $\beta$-arrestin 2 mediates $\beta$-2 adrenergic receptor signaling inducing prostate cancer cell progression. Oncol Rep. 2011;26 (6):1471-1477.

16. Raghuwanshi SK, Nasser MW, Chen X, Strieter RM, Richardson RM. Depletion of beta-arrestin-2 promotes tumor growth and angiogenesis in a murine model of lung cancer. J Immunol. 2008;180 (8):5699-5706.

17. Pikman Y, et al. MPLW515L is a novel somatic activating mutation in myelofibrosis with myeloid metaplasia. PLoS Med. 2006;3 (7):e270.

18. Kleppe M, et al. JAK-STAT pathway activation in malignant and nonmalignant cells contributes to MPN pathogenesis and therapeutic response. Cancer Discov. 2015;5 (3):316-331.

19. Lapidot T, Dar A, Kollet O. How do stem cells find their way home? Blood. 2005;106(6):1901-1910.

20. Alekhina O, Marchese A. $\beta$-Arrestin1 and signal-transducing adaptor molecule 1 (stam1) cooperate to promote focal adhesion kinase autophosphorylation and chemotaxis via the chemokine receptor CXCR4. J Biol Chem. 2016;291(50):26083-26097.

21. Clift IC, Bamidele AO, Rodriguez-Ramirez C, Kremer KN, Hedin KE. $\beta$-Arrestin 1 and distinct CXCR4 structures are required for stromal derived factor-1 to downregulate CXCR4 cell-surface levels in neuroblastoma. Mol Pharmacol. $2014 ; 85$ (4):542-552

22. Torossian F, et al. CXCR7 participates in CXCL12-induced CD34+ cell cycling through $\beta$-arrestin-dependent Akt activation. Blood. 2014;123 (2):191-202.

23. Sun Y, Cheng Z, Ma L, Pei G. Beta-arrestin2 is critically involved in CXCR4-mediated chemotaxis, and this is mediated by its enhancement of p38 MAPK activation. J Biol Chem. 2002;277 (51):49212-49219.

24. Kim J, et al. Beta-arrestins regulate atherosclerosis and neointimal hyperplasia by controlling smooth muscle cell proliferation and migration. Circ Res. 2008;103 (1):70-79.

25. Liu Z, et al. $\beta$-Arrestin-2 modulates radiation-induced intestinal crypt progenitor/stem cell injury. Cell Death Differ. $2016 ; 23$ (9):1529-1541.

26. Ahn S, Kim J, Hara MR, Ren XR, Lefkowitz RJ. \{beta\}-Arrestin-2 mediates anti-apoptotic signaling through regulation of BAD phosphorylation. J Biol Chem. 2009;284 (13):8855-8865.

27. Vermes I, Haanen C, Steffens-Nakken H, Reutelingsperger C. A novel assay for apoptosis. Flow cytometric detection of phosphatidylserine expression on early apoptotic cells using fluorescein labelled Annexin V. J Immunol Methods. 1995;184(1):39-51

28. Chen $\mathrm{M}$, et al. Genetic deletion of $\beta$-arrestin-2 and the mitigation of established airway hyperresponsiveness in a murine asthma model. Am J Respir Cell Mol Biol. 2015;53 (3):346-354.

29. Verstovsek S, et al. A double-blind, placebo-controlled trial of ruxolitinib for myelofibrosis. NEngl J Med. 2012;366(9):799-807.

30. Tefferi A. Challenges facing JAK inhibitor therapy for myeloproliferative neoplasms. N Engl J Med. 2012 ;366 (9):844-846.

31. Sun WY, et al. Down-regulation of $\beta$-arrestin2 promotes tumour invasion and indicates poor prognosis of hepatocellular carcinoma. Sci Rep. 2016;6:35609.

32. Jing $X$, et al. $\beta$-arrestin 2 is associated with multidrug resistance in breast cancer cells through regulating MDR1 gene expression. Int J Clin Exp Pathol. 2015;8 (2):1354-1363.

33. Duan $X$, et al. $\beta$-Arrestin 2 contributes to cell viability and proliferation via the down-regulation of FOXO1 in castration-resistant prostate cancer. J Cell Physiol. 2015;230 (10):2371-2381.

34. Alemayehu $\mathrm{M}$, et al. $\beta$-Arrestin2 regulates lysophosphatidic acid-induced human breast tumor cell migration and invasion via Rap1 and IQGAP1. PLoS One. 2013;8 (2):e56174.

35. Qin R, et al. $\beta$-Arrestin1 promotes the progression of chronic myeloid leukaemia by regulating BCR/ABL H4 acetylation. $B r J$ Cancer. 2014;111 (3):568-576.

36. Shu Y, et al. $\beta$-Arrestin1 promotes the self-renewal of the leukemia-initiating cell-enriched subpopulation in B-lineage acute lymphoblastic leukemia related to DNMT1 activity. Cancer Lett. 2015;357 (1):170-178.

37. Alekhina O, Marchese A. $\beta$-Arrestin1 and signal-transducing adaptor molecule 1 (STAM1) cooperate to promote focal adhesion kinase autophosphorylation and chemotaxis via the chemokine receptor CXCR4. J Biol Chem. 2016;291 (50):26083-26097.

38. McGovern KW, DeFea KA. Molecular mechanisms underlying beta-arrestin-dependent chemotaxis and actin-cytoskeletal reorganization. Handb Exp Pharmacol. 2014;219:341-359.

39. Hunton DL, et al. Beta-arrestin 2-dependent angiotensin II type 1A receptor-mediated pathway of chemotaxis. Mol Pharmacol. 2005;67 (4):1229-1236.

40. Hollingsworth JW, et al. Both hematopoietic-derived and non-hematopoietic-derived \{beta\}-arrestin-2 regulates murine allergic airway disease. Am J Respir Cell Mol Biol. 2010;43 (3):269-275.

41. Kotula JW, et al. Targeted disruption of $\beta$-arrestin 2-mediated signaling pathways by aptamer chimeras leads to inhibition of leukemic cell growth. PLoS One. 2014;9 (4):e93441.

42. Duncan AW, et al. Integration of Notch and Wnt signaling in hematopoietic stem cell maintenance. Nat Immunol. 2005;6 (3):314-322

43. Domen J, Cheshier SH, Weissman IL. The role of apoptosis in the regulation of hematopoietic stem cells: Overexpression of Bcl-2 increases both their number and repopulation potential. J Exp Med. 2000;191 (2):253-264.

44. Mannucci PM, Bettega D, Chantarangkul V, Tripodi A, Sacchini V, Veronesi U. Effect of tamoxifen on measurements of hemostasis in healthy women. Arch Intern Med. 1996;156 (16):1806-1810.

45. Forsberg JG. Estrogen effects on lymphoid tissue in neonatal and adult female mice. Acta Anat (Basel). 1995;153 (1):20-30. 46. Ho PC, Tang GW, Lawton JW. Lymphocyte subsets in patients with oestrogen deficiency. J Reprod Immunol. 1991;20 (1):85-91. 47. Peppel K, Jacobson A, Huang X, Murray JP, Oppermann M, Freedman NJ. Overexpression of G protein-coupled receptor 
kinase-2 in smooth muscle cells attenuates mitogenic signaling via $G$ protein-coupled and platelet-derived growth factor receptors. Circulation. 2000;102 (7):793-799.

48. Kim J, Ahn S, Rajagopal K, Lefkowitz RJ. Independent beta-arrestin2 and Gq/protein kinase Czeta pathways for ERK stimulated by angiotensin type $1 \mathrm{~A}$ receptors in vascular smooth muscle cells converge on transactivation of the epidermal growth factor receptor. J Biol Chem. 2009;284 (18):11953-11962.

49. Attramadal H, et al. Beta-arrestin2, a novel member of the arrestin/beta-arrestin gene family. J Biol Chem. 1992;267 (25):17882-17890.

50. Sipkins DA, et al. In vivo imaging of specialized bone marrow endothelial microdomains for tumour engraftment. Nature. 2005;435 (7044):969-973. 